\title{
Using object-based image analysis to map commercial poultry operations from high resolution imagery to support animal health outbreaks and events
}

\author{
Susan Maroney, ${ }^{1}$ MaryJane McCool-Eye, ${ }^{2}$ Andrew Fox, ${ }^{2}$ Christopher L. Burdett ${ }^{1}$ \\ ${ }^{1}$ Department of Biology, Colorado State University; ${ }^{2}$ Veterinary Services, Center for Epidemiology \\ and Animal Health, USDA APHIS, Fort Collins, CO, USA
}

\begin{abstract}
Precise locations of commercial poultry operations are important to planning and response for animal health outbreaks and events. These data are not available nationally or uniformly in the United States. This project uses machine learning capabilities to identify and map commercial poultry operations from aerial imagery in seven south-eastern states in the United States. The output protocol uses an Object-Based Image Analysis (OBIA) approach, which identifies objects based on spectral signatures combined with spatial, contextual, and textural information. The protocol is a semi-automated and user-assisted process, meaning that the object identification routines require minimal user inputs or expertise. Using the protocol, we produced locations of likely commercial poultry operations in up to two counties in one workday, about two times faster than manual digitisation. The resulting datasets provide an estimate of the number and geographic distribution of commercial poultry operations to assist outbreak response by augmenting available knowledge in affected areas.
\end{abstract}

Correspondence: Susan Maroney, Colorado State University, Department of Biology, 1878 Campus Delivery, Fort Collins, 80523 CO, USA

Tel.: +1.9704947335.

E-mail: susan.a.maroney@usda.gov

Key words: OBIA; machine learning; USDA; Feature Analyst; poultry.

Acknowledgements: This work was performed under APHIS/VS/ CEAH Cooperative Agreement Number 16-9208-0465-CA, CSU Cooperative Agreement Number 53-71151 with the U.S. Department of Agriculture and Colorado State University respectively. The authors thank Chris Kizer, Chris McCarty, and David South for their role in reviewing the OBIA protocol.

Conflict of interests: The authors declare no potential conflict of interests.

Received for publication: 7 July 2020.

Accepted for publication: 6 August 2020.

CCopyright: the Author(s), 2020

Licensee PAGEPress, Italy

Geospatial Health 2020; 15:919

doi:10.4081/gh.2020.919

This article is distributed under the terms of the Creative Commons Attribution Noncommercial License (CC BY-NC 4.0) which permits any noncommercial use, distribution, and reproduction in any medium, provided the original author(s) and source are credited.

\section{Introduction}

Infectious diseases in farmed animal populations have major economic implications on United States (U.S.) food systems and global trade. For example, a recent U.S. outbreak of Highly Pathogenic Avian Influenza (HPAI), a zoonotic disease that infects both animals and humans, required the depopulation of almost 50 million birds in the poultry industry, with the cost of control measures exceeding $\$ 870$ million (Johnson et al., 2016). Commercial poultry farm locations are an important dataset used in preventing the introduction and spread of disease. However, the only comprehensive national dataset of poultry operations in the United States is the United States Department of Agriculture (USDA), National Agricultural Statistics Service (NASS), Census of Agriculture (CoA), which is conducted every five years. United States law requires information from individual census respondents to remain confidential, so NASS publishes aggregated data by state and by county only if there are sufficient respondents in a county to avoid identifying individual or farm-specific data.

Other possible means to acquire locations of poultry operations include manual digitisation of aerial imagery. Manual digitisation methods allow actual operation locations to be identified by visual inspection of aerial imagery. Manual identification is timeconsuming; however, for small outbreaks it may be appropriate. As the size of the area affected increases, this manual process quickly becomes impractical.

Additionally, models such as the Farm Location and Agricultural Production Simulator (FLAPS) have been developed to simulate locations of farms, however they do not output actual farm locations. These models are intended for coarse estimation of farm size and location and are used for planning and research (Burdett et al., 2015). A need exists to rapidly identify commercial poultry barns over large areas for efficient planning and response to poultry health events and outbreaks.

A faster and more efficient method is presented here using Object-Based Image Analysis (OBIA) software to identify commercial poultry barns in satellite or aerial imagery and map their locations. This OBIA approach is feasible for large geographic areas due to the increasing availability of high resolution remotely-sensed imagery and faster processing speeds to handle this imagery (Blaschke et al., 2000). The OBIA approach works by identifying objects based on their spectral signatures combined with spatial, contextual, and textural information (Blaschke, 2010).

Previously, OBIA for building identification was applied to high-density settings like urban areas or refugee camps (Freire et al., 2014; Spröhnle et al., 2014). Identification of individual buildings from imagery has focused on small geographic areas (Caggiano et al., 2016). Software companies incorporated OBIA tools into their commercial software programs allowing identifica- 
tion of buildings in natural and human-made settings and processing of large geographic areas. Programs including the Feature Analyst extension for ArcGIS (Textron Systems, Providence, Rhode Island), eCognition (Trimble, Westminster, Colorado), and the ENVI Feature Extraction Module (by Harris Geospatial Solutions, Broomfield, Colorado). These programs combine various Machine Learning (ML) algorithms in an ensemble model, a technique which often produces a more accurate result than any one learning algorithm run individually (Opitz and Blundell, 2008; Seni and Elder, 2010).

For many years, OBIA has been employed for land cover classification. For example, the United States Forest Service used Feature Analyst to identify clumps of invasive Russian olive trees from aerial photography in the Fishlake National Forest in Utah (Hamilton et al., 2006). In Wake County, North Carolina, classification of a landscape into pervious and impervious surfaces allowed assessment of the effect of growing impervious surface area on aquatic fauna (Miller et al., 2009). More recently, OBIA has been applied for feature extraction in urban environments. In Lisbon, Portugal, Feature Analyst was used to extract urban buildings to update municipal maps and databases (Freire et al., 2014). In addition, OBIA was used to identify non-traditional buildings at a refugee camp in Somalia. Data about the number of these structures combined with an assumed density of people per square meter allowed aid workers to size relief resources for the camp's inhabitants (Spröhnle et al., 2014). In another example, analysts created a process for mapping human-made structures that would need protection in the event of wildland fires (Caggiano et al., 2016).

For this study, the objective was to develop a protocol that uses a repeatable, semi-automated, user-assisted ML process to identify and map commercial poultry barns from aerial imagery across a variety of poultry production types in the United States. The protocol identified locations of commercial poultry operations in up to two counties in one day. It is semi-automated and user-assisted, meaning the object identification routine runs with minimal user inputs. A well-documented workflow allows a GIS technician to implement it in any county in the United States with little or no specialised training. The resulting dataset of locations provide an accurate estimate of the number and distribution of commercial poultry operations facilitating rapid planning and response during disease outbreaks.

\section{Materials and methods}

\section{Study area and imagery}

The project's study area consisted of clusters of three or four counties in areas with large commercial poultry populations (USDA-NASS-CoA, 2015b). We chose five southern states with the highest poultry sales in 2012 (Figure 1): Alabama, Arkansas, Georgia, Mississippi, and North Carolina (USDA-NASS-CoA, 2015a). For testing the OBIA protocol broadly, these five states provided diversity in ecosystems, poultry operation sizes, and poultry production types (e.g., broilers, layers, pullets, and turkeys). We ran the protocol in three counties in Louisiana, a state with a smaller commercial poultry industry to test it in areas with lower farm densities. In addition, during the project's development, a HPAI outbreak in two states (Tennessee and Alabama) provided an opportunity to run the protocol in an emergency response situation. While incident data were not available for validation, the protocol's speed and ease of use were tested.

We used three-band, true-colour National Agriculture Imagery Program (NAIP) aerial imagery gathered between 2014 and 2016 (USDA-FSA, 2019). The 1-m resolution provided sufficient detail to detect an individual poultry barn. An advantage to using NAIP imagery is that it is published at a county-level as Compressed County Mosaics (USDA-NRCS, 2020), which makes downloading easy and mosaicking unnecessary.

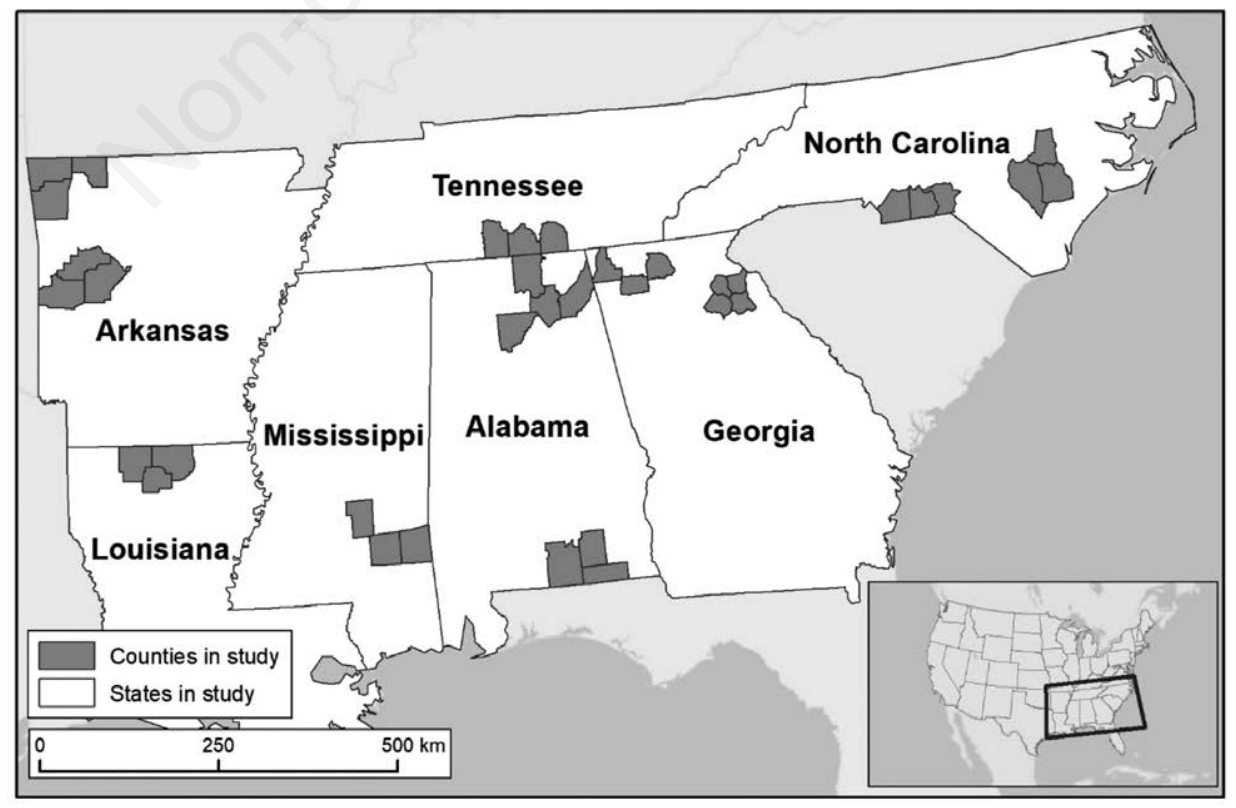

Figure 1 . The study area included 11 clusters of counties across seven states. 


\section{Object-based image analysis protocol}

We used the Feature Analyst extension (Overwatch Systems, 2010b) for ArcGIS (Environmental Systems Research Institute, 2016) to extract commercial poultry barns from the NAIP imagery. Feature Analyst uses a supervised ML algorithm to identify objects in imagery, driven by user-defined samples and inputs. (Blundell et al., 2008). This supervised learning routine requires the analyst to develop a training data set and select an input pattern (Figure 2). For the training set, an analyst selects features in the imagery that represent the range of the target objects, including size, shape and spectral signature. An analyst then chooses an input pattern that reflects the type of object (e.g., linear, land cover, building). Feature Analyst provides a list of input patterns and suggests choices for given types of target objects (Overwatch Systems, 2010a). Based on these inputs, the Feature Analyst extension decides on which ML algorithms to apply and combines them in an ensemble model. Candidate algorithms in Feature Analyst include artificial neural networks, decision trees, Bayesian learning, and K-nearest neighbor (Opitz and Blundell, 2008). The extension knows which algorithms have proven successful in identifying similar objects and with similar imagery. After examining the results of the first pass, an analyst can select examples of correct and incorrect features to refine the training set and repeat the processing to improve the results. (Opitz and Blundell, 2008). When Feature Analyst runs, it creates a Feature Model of the process including references to the imagery used, the training samples, the input pattern, the supervised learning algorithm, and the retraining steps (Figure 2). In addition, the model also contains the automated ML algorithms run during the procedure. The model can be applied to other images using the Batch Processing capability in Feature Analyst.

\section{Inputs}

Resampling each county's NAIP imagery from a $1-\mathrm{m}$ to $2-\mathrm{m}$ resolution sped up processing while still providing enough detail to identify poultry barns. Then, we reviewed the imagery of the county and digitized barns as training samples which reflected a range of barn sizes and roof spectral signatures. The regular rectangular shape and consistent spectral signature of the typical metal roofs of poultry barns provided distinctive targets for identification in imagery. We selected an input pattern that works well for detecting large residential and commercial buildings (Overwatch Systems, 2010a).

\section{Supervised learning and filtering features by geometry}

After defining these inputs, we ran Feature Analyst's OBIA routine. The output was a vector-based file of polygon features. This output included false positive features like strips of motorway and square buildings. The next step filtered these results using geometric criteria. The range of dimensions of typical barns used by any production type in the poultry industry are $90-183 \mathrm{~m}$ in length by $12-20 \mathrm{~m}$ in width (Figure 3; Bell and Weaver, 2002; Fairchild, 2005). Length, width, and length/width ratio fields were computed for each feature.

Some output features captured only part of the barn roof or included bare ground around the barn. In order to retain these valid features, we defined the range of features to delete as the typical dimensions stated above, plus a buffer. Features with lengths of less than $50 \mathrm{~m}$ or greater than $200 \mathrm{~m}$ were deleted (Figure 4). Also, as shown in Figure 4, commercial poultry barns tend to be a rectangular shape with a long length and short width. Given that the typical length/width ratio of a barn is between 2.0 and 10.0, all features not meeting this shape criteria were deleted.

\section{Retraining and removing false positives}

The output feature set still included false positives like features that were not buildings, for example, sand bars on rivers, and patches of bare ground. The next step was to eliminate additional false positives from the results using Feature Analyst's Remove Clutter by Shape tool. This tool improved the next iteration of the learning algorithm by analysing correct and incorrect example features and refining the learning model of the target objects (Figure 5). The tool repeated the classification deleting features that did not conform to the target objects. Of the options in the Remove Clutter by Shape tool, three were the most useful for gathering information about the shapes of buildings: Area, Second Order Moments, and Invariants. We applied these options in one iteration of the Remove Cluster by Shape tool because together they identified and deleted features that fell outside the limits of area, shape, and orientation expected of commercial poultry barns.

The final step of the OBIA protocol converted the barn features to operation points. Barn centroid points within $100 \mathrm{~m}$ of one another were combined into one operation (Figure 6).

After this step, the results for a county consisted of a few hundred points which we manually validated by visually inspecting each point on top of the aerial image. Additional false positives

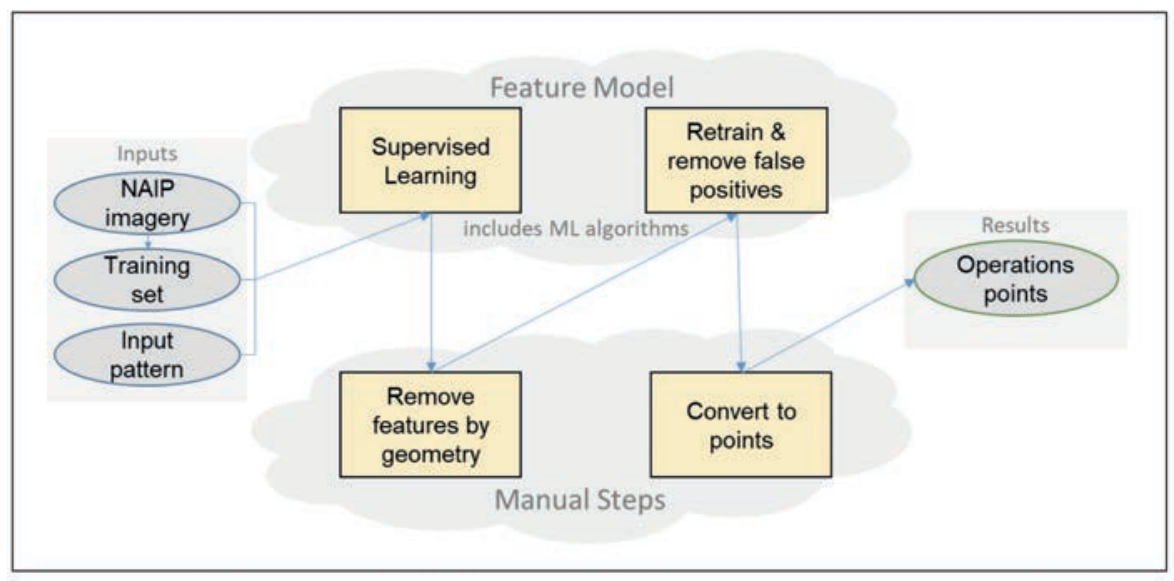

Figure 2. The protocol consisted of steps using the Feature Analyst extension plus manual steps to identify commercial poultry operations. 
were visually identified and manually removed, and missing operations not captured by the protocol were manually added to the results. The final output was a geodatabase for each state containing results of all the counties we processed, as well as a state model that can be applied to any other counties in the state.

\section{Time-cost estimates}

A key objective of this study was to develop a semi-automated protocol that was faster than manual barn identification. Three GIS analysts tested the protocol during development. Each analyst applied the protocol to a county in the study area. Each analyst recorded the time each step took and the number of features it produced. These statistics were added to those gathered by the lead author as input to a calculation for estimating the time-cost of running the protocol. We then explored the influence of various factors on the time required to complete our automated barn-identification process. First, we transformed all variables by their natural logarithm. Second, we examined, at a county level, the correlation between the number of barns detected and the number of operations reported by the CoA. Finally, we created several candidate
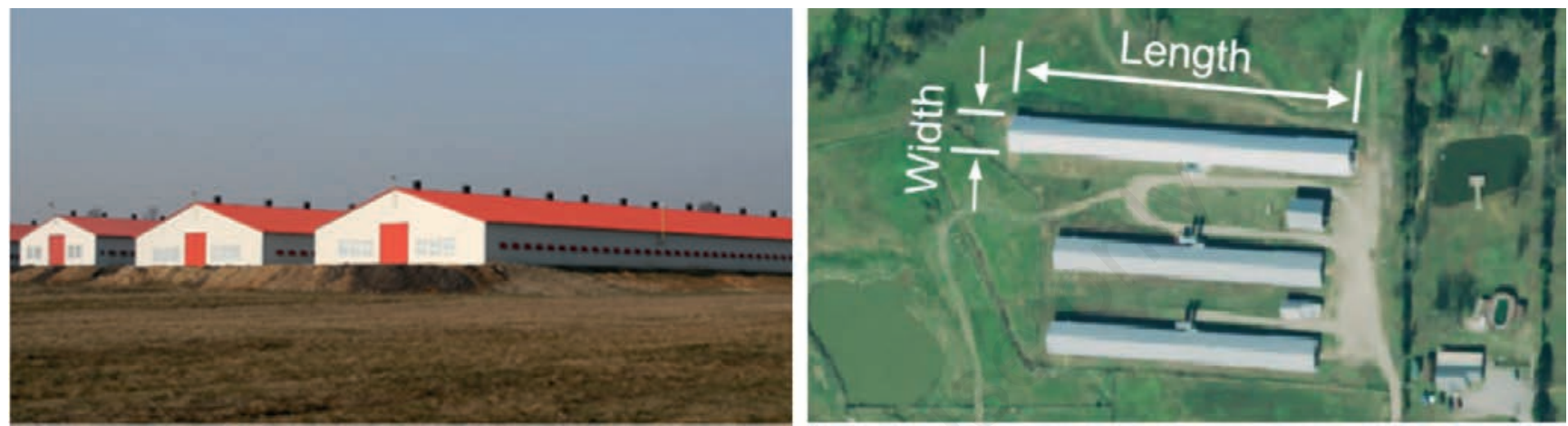

Figure 3. Aerial views of typical commercial poultry barns are shown with dimensions labelled for use in filtering OBIA results.

\begin{tabular}{|c|c|c|c|}
\hline \multicolumn{2}{|c|}{ (a) $50 \mathrm{~m}<$ Length $<200 \mathrm{~m}$} & \multicolumn{2}{c|}{ (b) $2.0<$ Length/Width ratio $<10.0$} \\
\hline Length $=50.53 \mathrm{~m}$ & Length $=166.2 \mathrm{~m}$ & $\mathrm{~L} / \mathrm{W}=38 / 28=1.4$ & $\mathrm{~L} / \mathrm{W}=286 / 12=23.8$ \\
Barn retained & Bam retained & Building deleted & Road segment deleted \\
\hline & & & \\
\hline
\end{tabular}

Figure 4. Panel (a) shows features that met the length criteria of a commercial poultry barn and were retained. Panel (b) shows features that violated the length-to-width ratio and were deleted.

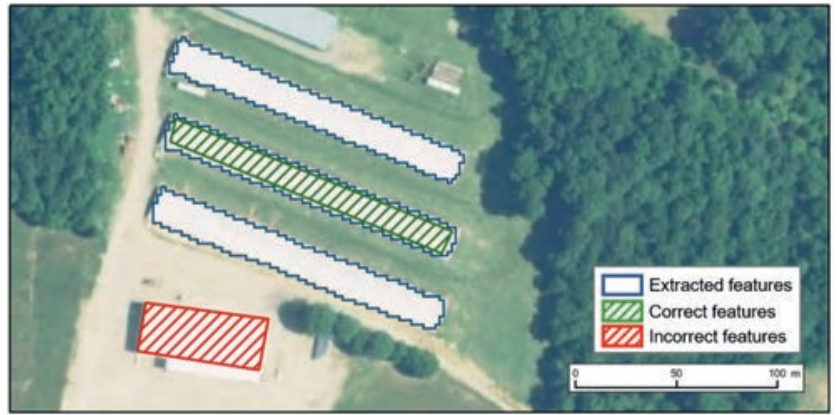

Figure 5. Correctly and incorrectly identified features were digitised to retrain the results.

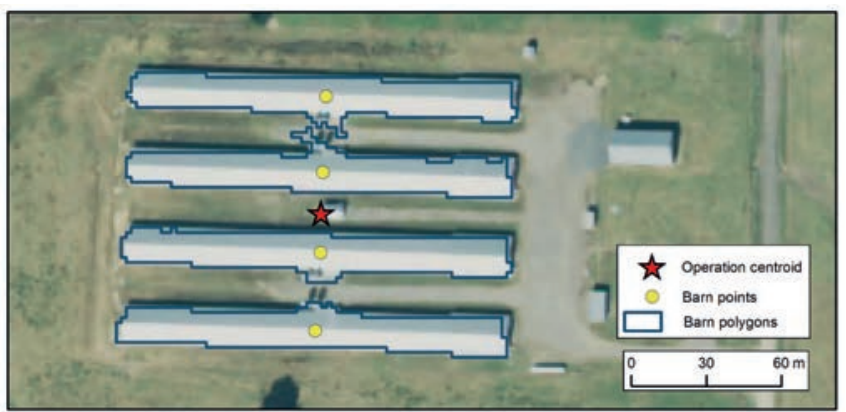

Figure 6. An example of the protocol's results - identifying polygons for individual barns, point locations for individual barns, and a single point (centroid) for the operation. 
models and used simple and multiple linear regressions to explore the relationship between processing time and three covariates: i) the number of barns detected during the process; ii) the area of the county; and iii) the number of operations reported for the county in the CoA. We evaluated our candidate models with informationtheoretic inference, ranking them by their Akaike Information Criterion (AIC) and $\triangle \mathrm{AIC}$ values.

\section{Model validation}

We performed an accuracy assessment on a subset of the study area using the best available ground truth data to validate the output of our OBIA protocol. We obtained validation datasets of known locations of poultry operations for three counties in North Carolina (Anson, Richmond, and Union counties) and three counties in Arkansas (Benton, Carroll, and Washington counties). For North Carolina, the validation dataset used was the state's Light Detection and Ranging (LiDAR) building-footprint dataset (NCFMP, 2018). We identified poultry barns from this LIDAR data using the same constraints on the length and the length/width ratio used in the protocol, followed by visual inspection by an analyst. The Arkansas GIS Office (AGO) provided the poultry barn validation dataset from their GeoStor dataset (AGO, 2014), which we manually updated, adding or deleting barns based on recent imagery. In both states, we aggregated clusters of barns within 100 $\mathrm{m}$ of one another into individual operations. These validation datasets served as our expected output, and the protocol's final dataset served as our observed output.

We used a $500-\mathrm{m}$ grid to define congruence between our expected and observed datasets. We found that commercial poultry operations were greater than $1 \mathrm{~km}$ from adjacent operations. This grid size minimized the chance of multiple operations occurring within a single grid cell. We first overlaid the expected dataset with the 500-m grid in ArcGIS to identify those grid cells that contained a known poultry operation. Cells containing an actual poultry operation defined the number of Positive cases (P), whereas cells that did not contain an actual poultry operation defined the number of
Negative cases $(\mathrm{N})$. We then performed the same procedure with our observed dataset. Lastly, we used ArcGIS to relate the number of positive and negative cases between both the expected and observed datasets and defined values for the number of True Positives (TP), True Negatives (TN), False Positives (FP), and False Negatives (FN), (Table 1). We used the values for P, N, TP, $\mathrm{TN}, \mathrm{FP}$, and FN in a confusion (or error) matrix to quantify several derived values to assess the accuracy of our modelled output predicting the location of poultry operations. The derived values included: i) sensitivity, or true positive value, ii) specificity, or true negative value, iii) precision or positive predictive value, iv) negative predictive value, v) accuracy, vi) quality index, vii) omission rate, viii) commission rate, and ix) commission error.

\section{Results}

\section{Study area}

Using the OBIA protocol, we successfully identified and mapped commercial poultry operations from 35 poultry-intensive counties in seven south-eastern states (Figure 7). Commercial poultry operations were clearly identifiable on the landscape. An

Table 1. Positive and Negative grid cells are defined and they relate in four possible ways from overlaying the expected and observed datasets.

\begin{tabular}{lll} 
Results & Name & Description \\
P & Positive & Grid cell contains an actual poultry operation \\
N & Negative & Grid cell does not contain an actual poultry operation \\
\hline TP & True Positive & An expected positive that is also an observed positive \\
TN & True Negative & An expected negative that is also an observed negative \\
FP & False Positive & An expected negative that is an observed positive \\
FN & False Negative & An expected positive that is an observed negative
\end{tabular}

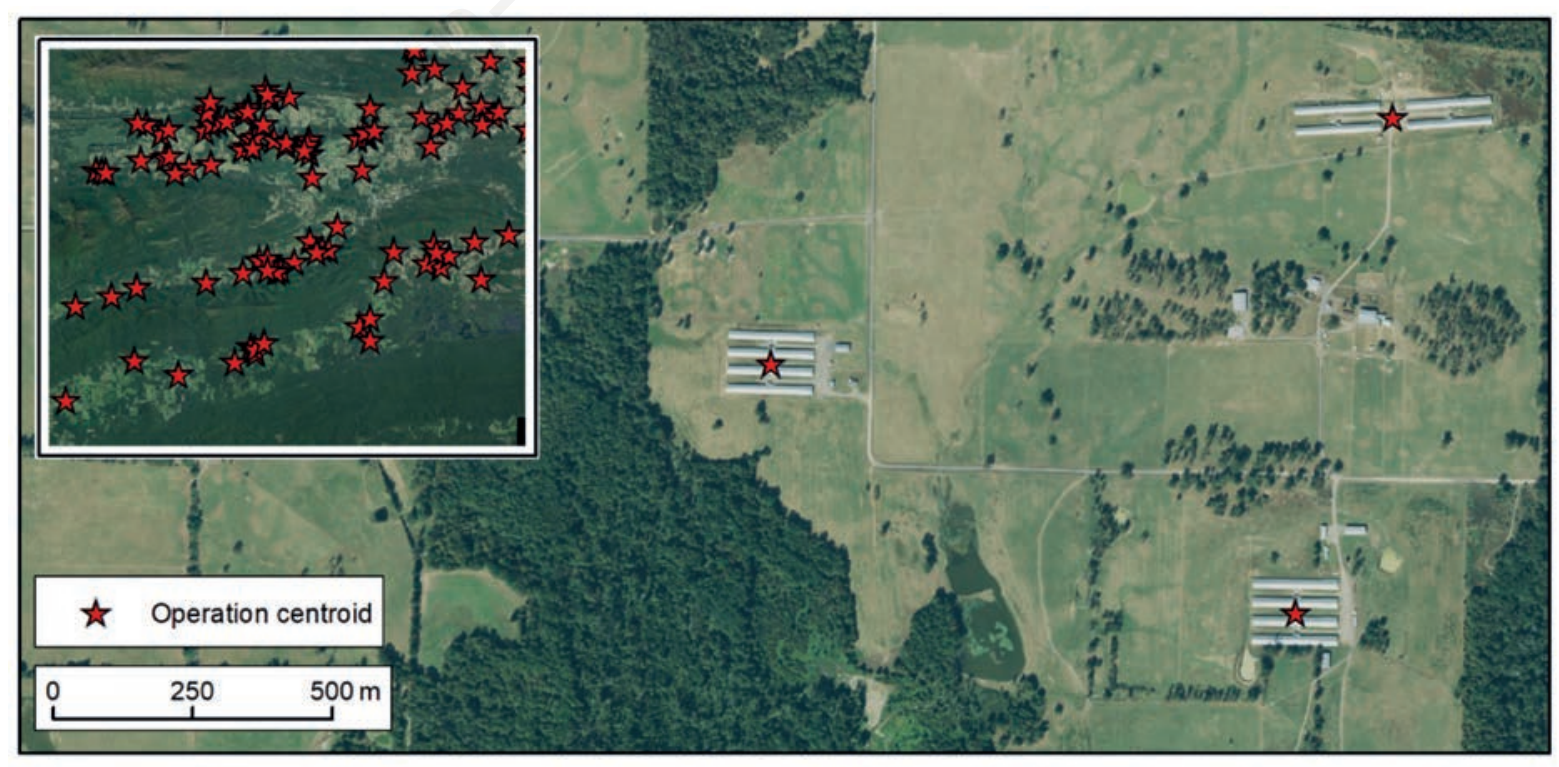

Figure 7. The results of the protocol are shown here for one of the 35 counties in the project. The overview map shows many of the operations identified by OBIA and the main map shows the accuracy of the locations for three separate operations. 
individual operation often consisted of multiple barns (from two to more than 20), although operations with single barns also occured. The stars represented the centroids of the operations. The inset map shows the distribution of operations in a portion of a county. The specific county shown is not identifed to preserve the privacy of individual operations.

Figure 8 displays the number of operations successfully identified in all seven states in the study. For each state Table 2 lists the number of counties processed using the protocol and the number of operations identified in those counties. In comparison, the CoA lists larger numbers of operations in these counties. The reason is that the CoA reports on operations of all sizes while this project focused on commercial operations comprised of large buildings that are readily identifiable in remotely sensed imagery. These large buildings house tens of thousands of birds at one time and so the locations of commercial operations provide valuable information on the concentration of birds across a response area.

\section{Object-based image analysis protocol}

The outputs of this project were two-fold: i) the protocol for identifying and mapping commercial poultry barns from remotely sensed imagery, and ii) the dataset of locations of commercial poultry operations for the 35 counties in the study, including validation. The protocol is available to others to create a dataset of commercial poultry operations. The protocol is documented in a detailed step-by-step guide based on the Feature Analyst extension and core ArcGIS capabilities. A GIS technician can run the protocol for outbreaks or events with minimal input needed. The final dataset of commercial poultry operations in the study area is available to responders and analysts.

\section{Time-cost estimates}

During the development process, we compared the developer's time to the three GIS analysts' time to complete the protocol for one county. On average, the additional analysts ran the protocol in 8.3 hours per county on average for the reviewers vs. 6.2 hours for the developer.

Compared to manual digitisation, implementation of the protocol improved results by decreasing the time to complete a county. The mean time-cost of conducting the protocol was $0.24(+/-0.11$ $\mathrm{SD})$ minutes $/ \mathrm{km}^{2}$. This time-cost means a $1660 \mathrm{~km}^{2}$ county would take 398 minutes ( $6.6 \mathrm{hrs})$ to process. In contrast, it took an analyst on average eight hours to identify and digitise poultry barns in a $1660 \mathrm{~km}^{2}$ county. For the entire 35 -county study area $(58,282$ $\mathrm{km}^{2}$ ), the time required to implement the protocol was 233 hours versus 282 hours for the manual digitisation method (saving more than a workweek of time). It is worth noting that the protocol runs largely independently so the number of man-hours is actually far lower than using manual methods.

The number of operations recorded in the CoA for a particular county was the factor that best predicted processing time (Table 3 ). Because the number of operations reported in the CoA and the number of barns detected during the protocol were highly correlated $(r=0.67)$, we did not include these two variables in the same candidate model. Although it had a lower $\triangle \mathrm{AIC}$ score, the candidate model that included two factors (Model \#1, Table 3) is not considered a better model than the simpler model that only included the number of operations (Model \#2, Table 3). This is because including the effect of county area did not increase Model \#2's $\triangle \mathrm{AIC}$ value by $>2.0$ (Burnham and Anderson, 2002).

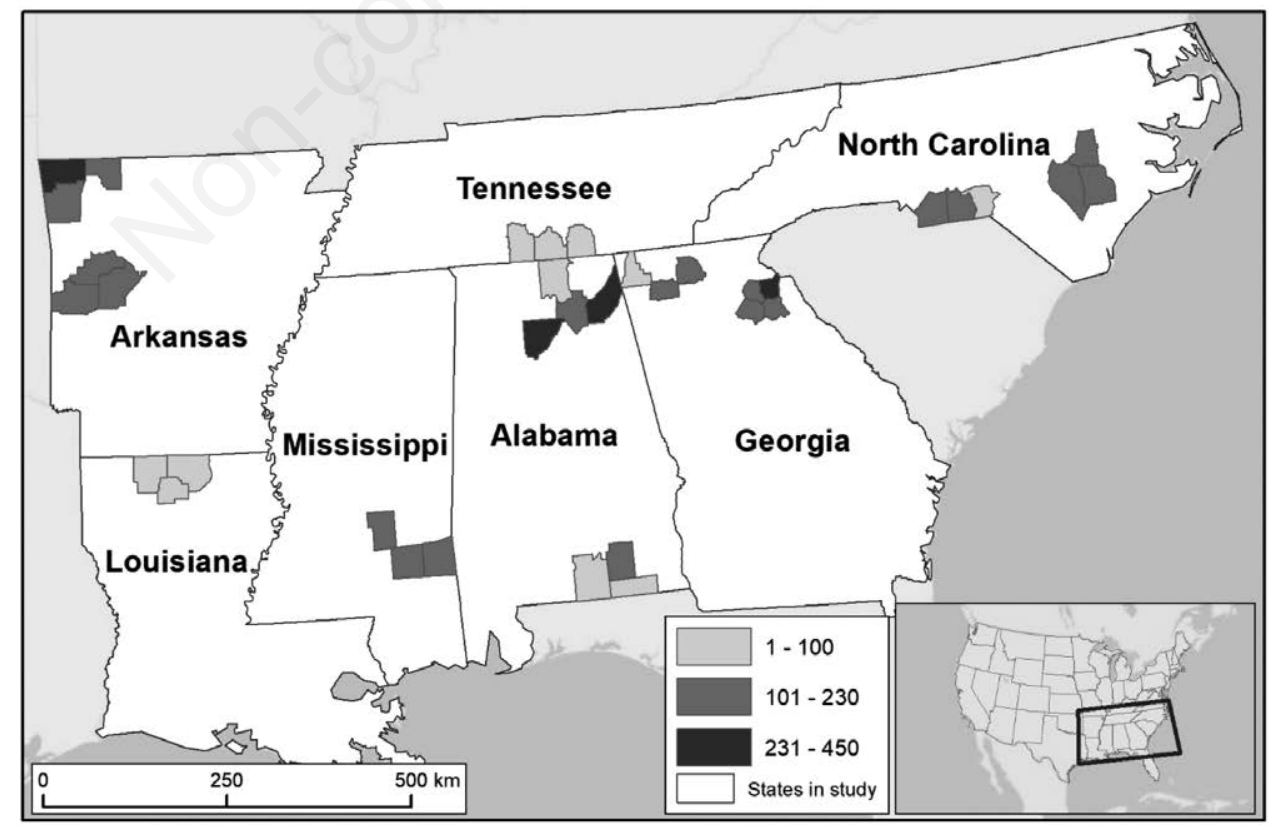

Figure 8. The number of commercial poultry operations identified by the OBIA protocol in the seven states in the study are displayed in three classes. 


\section{Model validation}

We used a confusion matrix to calculate metrics of accuracy such as accuracy, sensitivity, and specificity as shown in Table 4. We completed the calculations for the North Carolina cluster (Union, Anson, and Richmond counties) and the Arkansas cluster (Benton, Carroll, and Washington counties). Overall, we validated six of the $35(17 \%)$ counties in the project.

Our methodology to identify poultry barns has a mean accuracy rate of 0.983 with a higher mean value for negative predictive value (0.994) than positive predictive value (0.797), (Table 4$)$. The lowest values for both accuracy (0.948) and positive predictive value (0.552) were both obtained from Union County, North Carolina.

\section{Discussion}

We developed a protocol using commercial software that quickly generates locations of commercial poultry operations in a county to assist with response to an animal disease outbreak or animal health event. These locations assist outbreak responders by providing the locations of the population at immediate risk which subsequently improves targeting of surveillance, sizing resources for the response (people, materials, and equipment), and confirming locations of operations. Most importantly, these location data are not uniformly available from any other source for most of the United States. Model validation indicates the accuracy of our results were high, providing confidence in the value of the protocol and its applicability to other U.S. counties.

Table 2. The fraction of counties processed in each state and commercial poultry operations identified by the OBIA protocol.

\begin{tabular}{lcc}
$\begin{array}{l}\text { Totals for the study } \\
\text { State }\end{array}$ & $\begin{array}{c}\text { Number of counties processed/ } \\
\text { total number of counties }\end{array}$ & $\begin{array}{c}\text { Number of commercial } \\
\text { poultry operations mapped }\end{array}$ \\
Alabama & $7 / 67$ & 1294 \\
Arkansas & $6 / 75$ & 1076 \\
\hline Georgia & $7 / 159$ & 1070 \\
Louisiana & $3 / 64$ & 161 \\
\hline Mississippi & $3 / 82$ & 378 \\
North Carolina & $6 / 100$ & 924 \\
\hline Tennessee & $3 / 95$ & 82
\end{tabular}

Table 3. Information-theoretic inference and $R^{2}$ values for candidate models used to explore effect of various factors on processing time.

\begin{tabular}{llccc} 
Model & & $R^{2}$ & AIC & \\
1. & Number of operations * county area & 0.45 & 19.7 & 0 \\
2. & Number of operations & 0.35 & 21.6 & 1.9 \\
\hline 3. & Number of barns & 0.1 & 29.4 & 9.7 \\
4. & Number of barns * county area & 0.17 & 29.4 & 9.7 \\
\hline 5. & County area & 0.04 & 31 & 11.3 \\
\hline
\end{tabular}

Table 4. Results of the model validation show the metrics for North Carolina and Arkansas counties.

\begin{tabular}{|c|c|c|c|c|c|c|}
\hline \multirow[b]{2}{*}{ Value } & \multicolumn{3}{|c|}{ North Carolina } & \multicolumn{3}{|c|}{ Arkansas } \\
\hline & $\begin{array}{l}\text { Anson } \\
\text { County }\end{array}$ & $\begin{array}{l}\text { Richmond } \\
\text { County }\end{array}$ & $\begin{array}{l}\text { Union } \\
\text { County }\end{array}$ & $\begin{array}{l}\text { Benton } \\
\text { County }\end{array}$ & $\begin{array}{l}\text { Carroll } \\
\text { County }\end{array}$ & $\begin{array}{l}\text { Washington } \\
\text { County }\end{array}$ \\
\hline Sensitivity & 0.848 & 0.838 & 0.828 & 0.817 & 0.85 & 0.806 \\
\hline Specificity & 0.997 & 0.995 & 0.955 & 0.993 & 0.998 & 0.994 \\
\hline Positive Predictive Value & 0.881 & 0.786 & 0.552 & 0.827 & 0.951 & 0.786 \\
\hline Negative Predictive Value & 0.996 & 0.997 & 0.988 & 0.993 & 0.994 & 0.994 \\
\hline Accuracy & 0.992 & 0.992 & 0.948 & 0.987 & 0.992 & 0.988 \\
\hline Quality Index & 0.761 & 0.682 & 0.496 & 0.698 & 0.814 & 0.661 \\
\hline Omission Rate & 0.004 & 0.003 & 0.011 & 0.007 & 0.006 & 0.005 \\
\hline Commission Rate & 0.003 & 0.005 & 0.042 & 0.006 & 0.002 & 0.006 \\
\hline Commission Error & 0.003 & 0.005 & 0.045 & 0.007 & 0.002 & 0.006 \\
\hline
\end{tabular}


The protocol expanded on previous development in object identification and mapping in two respects. First, we used OBIA and ML to develop an operational tool for consistent and rapid use in emergency situations. At the time of this project, commercial software programs became available to run object identification by users rapidly and without requiring in-depth knowledge (Opitz and Blundell, 2008). Second, a review of the literature revealed that documented OBIA applications had typically been applied to small geographic areas or a small number of images (Freire et al., 2014; Caggiano, 2016). Applications to larger spatial extents such as demonstrated in this project were less common.

In the first case, the ability to produce a dataset of farm features rapidly enough to contribute to emergency operations was critical. Success hinged on exploiting the ML model within Feature Analyst (Overwatch Systems, 2010a). The scalability and robustness of the Feature Analyst software was demonstrated by our ability to create state models for poultry barn identification and apply it to 35 counties with a high level of accuracy. Finally, the protocol had to be sufficiently reliable and easy to use so that a GIS technician without specialised knowledge of the software, imagery, or the poultry industry could perform poultry barn identification in a new area (Opitz and Blundell, 2008).

In the second case, the ability to map poultry operations over large areas is also critical, because animal health emergency responses can include multiple counties or even states. Machine learning and the batch-processing capability in Feature Analyst allowed us to process up to 12 counties in one run (Overwatch Systems, 2010a). Larger spatial extents have become more common in OBIA studies. In one recent example, pinyon-juniper intrusions into the sagebrush ecosystem were classified in 6,230 digital orthophoto quarter quadrangles (approximately 228,000 $\mathrm{km}^{2}$ ) in Nevada and California (Gustafson et al., 2018). Another recent project created an automated process to identify possible concentrated animal feeding operations throughout North Carolina (Handan-Nader and Ho, 2019).

Machine learning approaches to object identification and mapping hold potential for continued improvement of animal-health applications. For the protocol, one future improvement could be attaching estimates of population size (i.e., the number of birds on an operation) based on the number of barns present on an operation. A second improvement could be implementing the protocol for other species (e.g., swine or cattle). A third improvement could be applying the protocol to other geographic regions or scaling it up to a national extent. In addition, LiDAR data could improve the building identification process. One potential advantage of using LiDAR over aerial imagery is the ability to use data fusion, which combines optical imagery with LiDAR or radar data. Tree canopy height from LiDAR combined with other types of data are already being used successfully in urban land-cover analysis (Zhang et al., 2017). In an object-based evaluation of dwellings in a refugee camp, optical data captured the dwelling footprints well, while the radar data separated contiguous dwellings (Spröhnle et al., 2017). Our experience using LiDAR data for our North Carolina validation dataset demonstrated that LiDAR has advantages over aerial imagery for automated detection of buildings with a characteristic size and shape. However, LiDAR is not yet collected in all parts of the United States and so not was not available for this project.

In addition to emergency applications, geospatial ML techniques can be adapted to improve the accuracy of planning and modelling tools. For example, combining simulated poultry demographic data with actual locations from OBIA could improve national poultry risk-modelling efforts or other emergency preparedness or surveillance planning.

\section{Conclusions}

Object-based image analysis applied to high resolution remotely sensed imagery produces precise location data for commercial poultry operations. The protocol in this project is built around OBIA. A GIS technician without programming or ML expertise can run the protocol easily. Thus, the protocol scales easily to respond quickly to an animal health emergency and provides locations of commercial poultry operations to augment any existing datasets. Using the protocol we developed, a GIS technician can process a county in 6.6 hours for an average-sized county in southeast United States, with an accuracy of $95-99 \%$. Based on the timecost estimates, implementing the protocol for the entire study area saved more than a workweek's time (49 hours) over the manual digitization method (233 hours versus 282 hours for the entire study area). One or two GIS technicians could run the protocol over that 233 hours, since only a small number of hours would be set-up and, for the bulk of the time, the process would run independently. In contrast, it would be challenging for some organisations to find the personnel with the GIS skills and knowledge of the poultry industry to digitise imagery for 282 hours. The time difference in receiving these geographic data on commercial poultry operations could be critical for emergency responders. Between the ease of use of the protocol, the processing time of 6.6 hours/county, and the confidence in the accuracy of the results, this protocol is a better option than manual digitisation for multiple counties. These data can provide a critical advantage to animal health emergency responders in a disease outbreak or other disaster when farm locations are scarce or unavailable.

\section{References}

AGO, 2014. Chicken House. Available from: https://gis.arkansas. gov/product/chicken-house-point/.

Bell Jr. DW, Weaver W (eds.), 2002. Commercial chicken meat and egg production, Kluwer Academic Publishers; 1265 pp.

Blaschke T, 2010. Object based image analysis for remote sensing. ISPRS J. Photogramm. Remote Sens 65:2-16.

Blaschke T, Lang S, Lorup E, Strobl J, Zeil P, 2000. Object-oriented Image Processing in an Integrated GIS/Remote Sensing Environment and Perspectives for Environmental Applications. Environ Inf Plan Politic 2:555-70.

Blundell S, Opitz D, Morris M, Rao R, 2008. Feature Analyst V5.0. ASPRS 2008 Annual Conference, 1-9. Available from: http://www.asprs.org/a/publications/proceedings/portland08/0 012.pdf

Burdett CL, Kraus B, Garza S, Miller R, Bjork K, 2015. Simulating the Distribution of Individual Livestock Farms and Their Populations in the United States: An Example Using Domestic Swine (Sus scrofa domesticus) Farms. PLoS One 10:e0140338.

Burnham KP, Anderson DR, 2002. Model Selection and Multimodel Inference. 2nd ed. Springer-Verlad, New York; $454 \mathrm{pp}$.

Caggiano M, Tinkham WT, Hoffman C, Cheng AS, Hawbaker TJ, 
2016. High resolution mapping of development in the wildland-urban interface using object based image extraction. Heliyon 2:e00174.

Environmental Systems Research Institute, 2016. ArcGIS Desktop. Redlands, CA. Available from: https://www.esri. com/en-us/arcgis/products/arcgis-desktop/overview

Fairchild BD, 2005. Basic Introduction to Broiler House Environmental Control. Available from: https://thepoultrysite.com/articles/basic-introduction-to-broiler-housingenvironmental-control Accessed: March 19, 2020.

Freire S, Santos T, Navarro A, Soares F, Silva JD, et al., 2014. Introducing mapping standards in the quality assessment of buildings extracted from very high resolution satellite imagery. ISPRS J. Photogramm Remote Sens 90:1-9.

Gustafson B, Coates PS, Roth CL, Chenaille MP, Ricca MA, et al., 2018. Using object-based image analysis to conduct high-resolution conifer extraction at regional spatial scales. Int J Appl Earth Observ Geoinform 73:148-55.

Hamilton R, Megown K, Lachowski H, Campbell R, 2006. Mapping Russian olive: Using remote sensing to map an invasive tree. U.S. Department of Agriculture, U.S. Forest Service, Remote Sensing Applications Center, RSAC-0087-RPT1. Available

from: https://www.researchgate.net/profile/Randy_Hamilton/publication/308783694_Mapping_Russian_olive_using_remote_se nsing_to_map_an_invasive_tree/links/57f0824508ae280dd0a eaa53/Mapping-Russian-olive-using-remote-sensing-to-mapan-invasive-tree.pdf?origin=publication_detail

Handan-Nader C, Ho DE, 2019. Deep learning to map concentrated animal feeding operations, Nat Sustain 2:298-306

Johnson KK, Seeger RM, Marsh TL, 2016. Local Economies and Highly Pathogenic Avian Influenza. Choices. Quarter 2. Available online: https://www.choicesmagazine.org/choicesmagazine/theme-articles/economic-consequences-of-highlypathogenic-avian-influenza/local-economies-and-highlypathogenic-avian-influenza

Miller JE, Nelson SAC, Hess GR, 2009. An Object Extraction Approach for Impervious Surface Classification with VeryHigh-Resolution Imagery. Prof Geogr 61:250-64.

North Carolina Floodplain Mapping Program (NCFMP), 2018. North Carolina's Spatial Data Download, Built Environment. Available from: https://sdd.nc.gov/

Overwatch Systems, Ltd., 2010a. Feature Analyst 5.0 for ArcGIS reference manual. Textron Systems, 354 pp. Available from: https://www.textronsystems.com/products/feature-analyst

Overwatch Systems, Ltd., 2010b. Feature Analyst extension. Sterling, VA. Available from: https://www.textronsystems. com/products/feature-analyst

Opitz D, Blundell S, 2008. Object recognition and image segmentation: the Feature Analyst approach. In: Blaschke T, Lang S, Hay GJ (eds.). Object-Based Image Analysis. Lecture Notes in Geoinformation and Cartography. Springer, Berlin, Heidelberg. doi:10.1007/978-3-540-77058-9_8

Seni G, Elder FL, 2010. Ensemble methods in data mining: Improving accuracy through combining predictions. Synthesis Lectures on Data Mining and Knowledge Discovery. Morgan and Claypool Publishers.

Spröhnle K, Fuchs E-M, Pelizari PA, 2017. Object-based analysis and fusion of optical and SAR satellite data for dwelling detection in refugee camps. IEEE J Sel Top Appl Earth Obs Remote Sens 10:1780-91. doi:10.1109/JSTARS.2017.2664982

Spröhnle K, Tiede D, Schoepfer E, Füreder P, Svanberg A, Rost T, 2014. Earth observation-based dwelling detection approaches in a highly complex refugee camp environment - a comparative study. Remote Sens 6:9277-97. doi:10.3390/rs6109277

United States Department of Agriculture, Farm Service Agency (USDA-FSA), 2019. NAIP Imagery. Available from: https://www.fsa.usda.gov/programs-and-services/aerial-photography/imagery-programs/naip-imagery/

United States Department of Agriculture, National Agricultural Statistics Service, Census of Agriculture (USDA-NASS-CoA), 2015a. 2012 Census of Agriculture Highlights: Poultry and Egg Production. Available from: https:/www.nass.usda.gov/ Publications/Highlights/2015/Poultry_and_Egg_Production.pdf

United States Department of Agriculture, National Agricultural Statistics Service, Census of Agriculture (USDA-NASS-CoA), 2015b. 2012 Census Volume 1, Chapter 2, County Level. Available from: https://www.nass.usda.gov/Publications/ AgCensus/2012/Full_Report/Volume_1,_Chapter_2_County_ Level/

United States Department of Agriculture, Natural Resources Conservation Service (USDA-NRCS), 2020. Geospatial Data Gateway, Direct NAIP Download. Available from: https://datagateway.nrcs.usda.gov/GDGHome_DirectDownLoad.aspx

Zhang C, Smith M, Fang C, 2017. Evaluation of Goddard's LiDAR, hyperspectral, and thermal data products for mapping urban land-cover types. GISci Remote Sens 55:1-20. doi:10.1080/15481603.2017.1364837 